\title{
Construção de um protocolo de restrição de movimento de coluna (RMC) vertebral: relato de experiência
}

\author{
Construction of a spinal motion restriction (SMR) protocol: experience report
}
Construcción de un protocolo de restricción del movimiento espinal (RMC): informe de experiencia

Paula Caroline Gonçales ${ }^{1 *}$, Leonardo Schiess Sales Antunes ${ }^{1}$, Daniela Aparecida Morais ${ }^{1}$, Cíntia Maria Guedes de Moraes, Ana Claudia Brito Costa Fernandes ${ }^{1}$, Karina Mara de Souza ${ }^{1}$.

\section{RESUMO}

Objetivo: Descrever a elaboração de um protocolo de restrição de movimento de coluna (RMC) vertebral do Serviço de Atendimento Móvel de Urgência de Belo Horizonte. Relato da experiência: Trata-se de um relato de experiência sobre a construção de um protocolo de RMC vertebral. Foi realizada a busca na literatura das melhores evidências sobre o tema, seguida de análise crítica dos estudos e a construção de um fluxograma de decisão para indicação ou não de RMC vertebral. Ficou definido que a RMC vertebral será realizada sempre que a equipe atender pacientes, vítimas de trauma, que apresentarem ao menos uma das seguintes características: instabilidade na avaliação primária, idade maior que 65 anos, presença de dor em coluna, déficit de consciência, déficit sensitivo ou motor, deformidade na coluna, distração, dinâmica do trauma significativa. Considerações finais: Ficou evidente a importância de se construir um protocolo clínico para guiar os profissionais aos melhores cuidados aos pacientes acometidos por trauma, assim como a necessidade de mudança de paradigmas com relação ao manejo destas vítimas e por fim a redução do tempo para seu tratamento definitivo.

Palavras-chave: Assistência pré-hospitalar, Imobilização, Coluna vertebral, Árvores de decisões.

\section{ABSTRACT}

Objective: To describe the development of a spinal motion restriction protocol (SMR) for the Mobile Emergency Care Service of Belo Horizonte. Experience report: This is an experience report on the construction of a vertebral SMR protocol. A search in the literature for the best evidence on the subject was performed, followed by a critical analysis of the studies and the construction of a decision flowchart for indicating or not vertebral SMR. It was defined that vertebral SMR will be performed whenever the team attends trauma victims who present at least one of the following characteristics: instability in the primary assessment, age over 65 years, presence of back pain, deficit of consciousness, deficit sensory or motor, spinal deformity, distraction, significant trauma dynamics. Final considerations: The importance of building a clinical protocol to guide professionals to better care for trauma patients was evident, as well as the need to change paradigms regarding the management of these victims and, finally, reduce the time for their treatment definitive.

Keywords: Pre-hospital care, Immobilization, Spine, Decision trees.

\section{RESUMEN}

Objetivo: Describir el desarrollo de un protocolo de restricción del movimiento espinal (CMR) para el Servicio Móvil de Atención de Emergencias de Belo Horizonte. Informe de experiencia: Este es un informe de experiencia sobre la construcción de un protocolo de RMC vertebral. Se realizó una búsqueda en la literatura de la mejor evidencia sobre el tema, seguida de un análisis crítico de los estudios y la construcción de un diagrama de flujo de decisiones para indicar o no RMC vertebral. Se definió que la RMC vertebral se realizará siempre que el equipo atienda a víctimas de trauma que presenten al menos una de las siguientes características: inestabilidad en la valoración primaria, edad mayor de 65 años, presencia de dolor lumbar, déficit de conciencia, déficit sensorial o motor, deformidad espinal, distracción, dinámica traumática significativa. Consideraciones finales: Se evidenció la importancia de construir un protocolo clínico que oriente a los profesionales hacia una mejor atención al paciente traumatizado, así como la necesidad de cambiar paradigmas en cuanto al manejo de estas víctimas y, finalmente, reducir el tiempo de su tratamiento definitivo.

Palabras clave: Atención prehospitalaria, Inmovilización, Columna vertebral, Árboles de decisión.

1 Serviço de Atendimento Móvel de Urgência (SAMU), Prefeitura de Belo Horizonte, Belo Horizonte - MG.

*E-mail: paulinha_sepulveda@yahoo.com.br 


\section{INTRODUÇÃO}

O atendimento ao politraumatizado no ambiente pré-hospitalar inclui a prevenção de lesões secundárias na medula espinhal através do transporte adequado. Desde 1960, diversos serviços médicos pré-hospitalares em todo mundo adotaram a estabilização de toda coluna do paciente usando um colar cervical e uma prancha rígida como a melhor estratégia para a redução do risco de lesões neurológicas. No entanto, o procedimento tem sido questionado nos últimos anos, devido à falta de estudos de qualidade que comprovem a sua eficácia (MASHAMANN C, et al., 2019).

Estudos demonstram que lesões cervicais são relativamente raras (2-4\%) e que dos pacientes com fratura de coluna cervical, somente cerca de $20 \%$ evoluíram com lesão medular (STIELL IG, et al., 2001; STIELL IG, et al., 2003; HASLER RM, et al., 2011; SUNDSTROM T, et al., 2014). As evidências também revelam os possíveis efeitos deletérios do uso da prancha rígida e do colar cervical para a imobilização da coluna vertebral como: dor, desenvolvimento de lesões por pressão, aumento da pressão intracraniana cerca de 4,5mmhg, risco de broncoaspiração, dificuldade de manejo da via aérea, aumento do tempo de permanência hospitalar, aumento do número de exames radiológicos e risco de deslocamento de fratura de coluna em pacientes idosos (STIELL IG, et al., 2001; STIELL IG, et al., 2003; PURVIS TA, et al., 2017; CONNOR D, et al., 2013; HAM WHW,et al., 2016; RAO JP, et al., 2016).

O uso da prancha rígida não reduz a movimentação da coluna vertebral pelo próprio formato da coluna em "S" e que a própria musculatura paravertebral do paciente estabiliza uma coluna potencialmente instável. Deste modo o termo "imobilização" não é o mais adequado a ser utilizado, pois, a imobilização total da coluna é quase impossível, e assim, houve a mudança para "restrição de movimento de coluna" (RMC) vertebral (FISCHER PE, et al., 2018; STANTON D, et al., 2017).

Embora a realização da RMC vertebral em pacientes traumatizados continue sendo parte integrante do atendimento pré-hospitalar, existem formas alternativas de se aplicar estas técnicas levando em consideração as evidências atuais com uso de protocolos baseados em regras de decisão (CONNOR D, et al., 2013). A RMC vertebral pode ser alcançada através da utilização de maca colher, de maca a vácuo, de maca da ambulância, ou outro dispositivo semelhante que ofereça as condições de segurança para o paciente (WHITE CC, et al., 2014; FISCHER PE, et al., 2018).

Sabe-se que uma porcentagem pequena de todas as vítimas de trauma contuso apresentarão lesão na coluna cervical e que estes estarão associados com a alteração do nível de consciência. Ressalta-se também que, nas vítimas conscientes e com avaliação confiável que apresentarem lesão vertebral, a própria musculatura paravertebral estabilizará a coluna e a utilização da imobilização padrão com prancha rígida poderá causar efeitos deletérios como já citado (SUNDSTROM T, et al., 2014; FISCHER PE, et al., 2018; STANTON D, et al., 2017).

Deste modo, diante das evidências e mudanças que vem ocorrendo mundialmente nos serviços de atendimento ao trauma, houve a necessidade por parte do Serviço de Atendimento Móvel de Urgência de Belo Horizonte (SAMU-BH) de iniciar as discussões a cerca da RMC vertebral, bem como de criar protocolo pautado nas evidências científicas e nas condições estruturais do serviço para que um atendimento de qualidade seja prestado a população. Assim, este estudo teve o objetivo de descrever a elaboração de um protocolo de restrição de movimento de coluna vertebral do Serviço de Atendimento Móvel de Urgência de Belo Horizonte.

\section{RELATO DE EXPERIÊNCIA}

O intuito deste relato é trazer para prática diária dos profissionais atuantes no pré-hospitalar móvel a importância da construção de protocolos para propiciar a equipe parâmetros, rotinas e cuidados baseados nas melhores evidências para os atendimentos das vítimas de trauma. A discussão de cada item do protocolo se deve aos cuidados evidenciados nos estudos selecionados para construção dele.

O SAMU-BH conta com 21 unidades de suporte básico e 7 unidades de suporte avançado, além de uma central de regulação médica das urgências. Possui cerca de 1.000 profissionais e realiza em média, 8.700 atendimentos por mês e até o presente momento, o protocolo institucional determina a imobilização tradicional para todas as vítimas de trauma. 
Foi levantada a necessidade de se discutir a RMC vertebral e para isso constituiu-se um grupo para estudar o tema. Este grupo realizou buscas nas bases de dados Web of Science, Cinahl, Medline, Scopus, Lilacs, utilizando as seguintes palavras-chave: "imobilização", "restrição de movimento de coluna", "assistência préhospitalar", "vítimas de trauma", "prancha rígida", "colar cervical", "coluna vertebral". Após a leitura e análise de artigos, foram selecionadas as melhores evidências e iniciou-se a construção do protocolo.

Observou-se que vários artigos indicaram que a escolha para se realizar a RMC vertebral fosse baseada em fluxogramas de tomada de decisão (STIELL IG, et al., 2001; VAILLANCOURT C, et al., 2009; STIELL IG, et al., 2003; KORNHALL DK, et al., 2017; MASHMANN C, et al., 2019).

Desta forma os autores optaram por construir um fluxograma de decisão objetivo, de fácil memorização e que atendesse as recomendações da literatura e do serviço, para orientar os profissionais quanto a realização ou não da RMC vertebral (Figura 1).

Figura 1 - Fluxograma de decisão para indicação de Restrição do Movimento de Coluna (RMC) vertebral do Serviço de Atendimento Móvel de Urgência de Belo Horizonte.

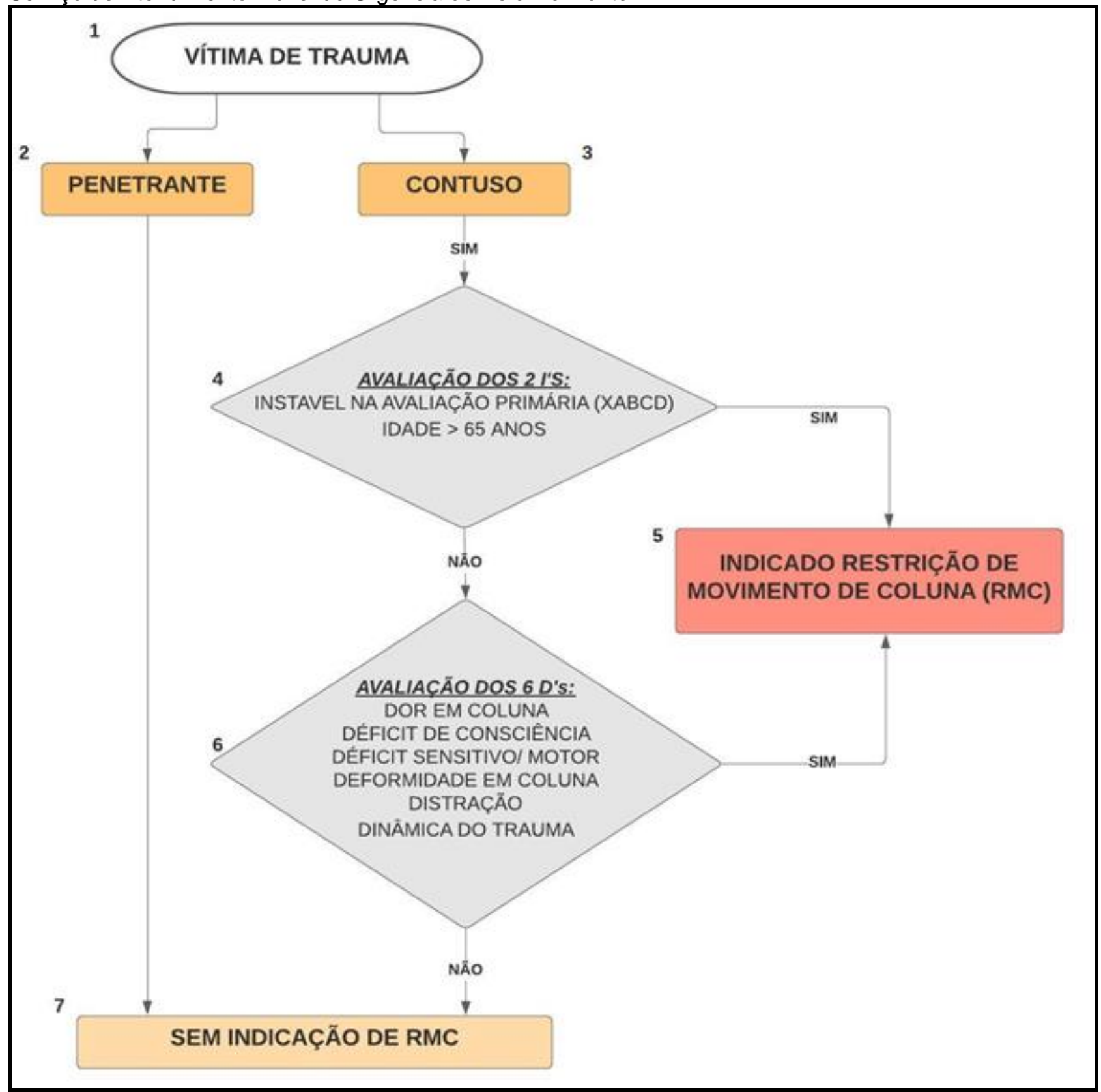

Fonte: Gonçales PC, et al., 2021. 
A caixa 1 do fluxograma mostra que pacientes vítimas de trauma podem ser divididos em dois tipos de trauma, o penetrante e o contuso. Observa-se que vítima de trauma penetrante (caixa 2) não tem indicação de RMC vertebral como mostra a caixa 7. Já para as vítimas com trauma contuso (caixa 3) é necessário seguir os quadros de decisão. Para isso os autores elaboraram um método mnemônico para memorização e rápido tratamento, o que chamaram de "Avaliação dos 2 l's (caixa 4) e 6 D's" (caixa 6), sendo que o "I" e o "D" significam as iniciais dos critérios avaliados, ou seja, "l" de instável na avaliação primária, de idade e "D" de dor na coluna, de déficit de consciência, de déficit sensitivo/motor, de deformidade de coluna, de distração e de dinâmica do trauma. Neste contexto se profissional diz "sim" para algum critério de decisão seguindo a sequência apresentada, realiza-se a RMC vertebral, se "não", passa para o próximo critério e segue a orientação definida no fluxograma.

Assim, a priori a equipe realizará avaliação primária, se houver presença de instabilidade realiza-se a RMC vertebral rapidamente (caixa 5) sem atrasar o tratamento das lesões encontradas. Se a vítima está estável e tem mais de 65 anos, também está indicado a RMC vertebral (caixa 5). Se estável e menor que 65 anos avalia os 6 D's, se positivo para algum critério realiza a RMC vertebral como mostrado na caixa 5, se negativo para os 6D's, não há indicação de se realizar a RMC vertebral conforme a caixa 7.

Os critérios de decisão, estabelecidos no fluxograma, foram selecionados a partir da avaliação das evidências associando os critérios de vários estudos e também considerando critérios estabelecidos pelo grupo de discussão levando em consideração a realidade do serviço (STIELL IG, et al., 2001; VAILLANCOURT C, et al., 2009; STIELL IG, et al., 2003; WHITE CC, et al., 2014; STANTON et al., 2017; KORNHALL DK, et al., 2017; MASHMANN C, et al., 2019). Para interpretação do fluxograma de decisão, o quadro a seguir descreve todos os significados dos termos contidos na figura 1 a fim de facilitar 0 entendimento dos profissionais durante sua utilização (Quadro 1).

Quadro 1 - Descrição dos critérios de decisão para indicar a Restrição do Movimento de Coluna (RMC) vertebral do Serviço de Atendimento Móvel de Urgência de Belo Horizonte.

\begin{tabular}{|c|c|}
\hline \multirow{2}{*}{\multicolumn{2}{|c|}{$\begin{array}{l}\text { CRITÉRIOS DE DECISÃO } \\
\text { AVALIACÃO DOS } 2 \text { I'S }\end{array}$}} \\
\hline & \\
\hline $\begin{array}{l}\text { Instável na avaliação } \\
\text { primária }\end{array}$ & $\begin{array}{l}\text { X- Presença de hemorragia externa exsanguinante; A- Risco ou via aérea } \\
\text { obstruída; B- Hipóxia, pneumotórax, hemotórax; C- Presença ou risco de } \\
\text { choque, D- Alteração do nível de consciência (diminuição na escala de coma } \\
\text { de Glasgow). }\end{array}$ \\
\hline \multirow{2}{*}{\multicolumn{2}{|c|}{ AVALIAÇÃO DOS 6 D'S }} \\
\hline & \\
\hline Dor em coluna & $\begin{array}{l}\text { Presença de dor referida ou a palpação de linha média da coluna vertebral; } \\
\text { dor a movimentação do pescoço em rotação ou flexão, observar sempre a } \\
\text { expressão facial. }\end{array}$ \\
\hline Déficit de consciência & $\begin{array}{l}\text { Escala de coma de Glasgow < 15; confuso; agitado; intoxicação por } \\
\text { álcool/drogas (fala arrastada, hálito etílico, perda de equilíbrio, agressividade } \\
\text { e/ou agitação e relato de testemunhas de uso de álcool e/ou drogas); } \\
\text { deficiência auditiva ou barreira linquística que impeca a comunicacão. }\end{array}$ \\
\hline $\begin{array}{l}\text { Déficit sensit } \\
\text { motor }\end{array}$ & $\begin{array}{l}\text { Presença de paresia, parestesia, plegia, formigamento, dormência, perda de } \\
\text { sensibilidade nos quatro membros. }\end{array}$ \\
\hline Deformidade em coluna & $\begin{array}{l}\text { Presença de deformidade em algum ponto da coluna desde a cervical até } \\
\text { lombossacra. }\end{array}$ \\
\hline Distração & $\begin{array}{l}\text { Presença de lesão e/ou dano que possa produzir dor suficiente causando } \\
\text { distração/confusão do paciente na avaliação de possível lesão de coluna } \\
\text { vertebral. Ex: lesão torácica, abdominal, musculoesquelética (fratura de } \\
\text { extremidades, lacerações, desluvamento, esmagamento), queimaduras, } \\
\text { perturbação emocional, outras lesões que prejudiquem a avaliação. }\end{array}$ \\
\hline Dinâmica do trauma & $\begin{array}{l}\text { Queda de altura maior que } 2 \text { metros; queda de no mínimo } 5 \text { degraus de } \\
\text { escada; trauma axial (Ex: mergulho, queda livre de objeto acima de } 2 \text { metros } \\
\text { sobre a vítima), vítima ejetada, colisão de bicicleta; atropelamento, } \\
\text { capotamento, cinemática de alta velocidade, qualquer outra situação de } \\
\text { trauma que considere importante a RMC vertebral. }\end{array}$ \\
\hline
\end{tabular}

Fonte: Gonçales PC, et al., 2021. 
Após a elaboração do fluxograma de decisão e do quadro de descrição dos critérios definiu-se como deveria ser a técnica de manipulação do paciente. Deste modo, optou-se por utilizar os protocolos institucionais a partir da posição em que a vítima é encontrada. Assim sempre que diante de uma vítima de trauma contuso, deverá realizar a estabilização manual da coluna cervical desde início do atendimento, até a indicação de RMC vertebral ou não, seguindo o fluxograma.

Toda vítima deve ser colocada em decúbito dorsal, utilizando a técnica de rolamento $90^{\circ}$ ou $180^{\circ}$. Nas situações que estiver com um capacete, a mesma conduta deve ser adotada e somente depois é que o capacete é retirado na técnica descrita em protocolo institucional (PHTLS, 2018). Após as ações citadas, se a RMC vertebral estiver indicada, deve-se utilizar o colar cervical e a prancha rígida, se o transporte até a unidade de destino for menor que 30 minutos. Ao chegar neste local, deve-se passar imediatamente para a maca retirando-a da prancha rígida, mantendo alinhamento do corpo e o colar cervical (CONNOR D, et al., 2013; STANTON D, et al., 2017; WHITE CC, et al., 2014; FISCHER PE, et al., 2018).

Uma outra alternativa preferencial, é realizar a RMC vertebral com a própria maca da ambulância e nesta situação, a prancha rígida é utilizada somente para a retirada da vítima da cena. Ao retirá-lo da prancha e colocá-lo na maca, deve-se manter o bashal na própria maca e um passante (lona de transferência de paciente, feito de lona lavável com alças laterais que facilitam a manipulação de uma maca a outra) podendo elevar a cabeceira em até 30ํㅡㄹ se necessário (CONNOR D, et al., 2013; STANTON D, et al., 2017; WHITE CC, et al., 2014; FISCHER PE, et al., 2018).

Em vítima estável e deambulando, sentada na cena e/ou automóvel, deve-se colocar o colar cervical, posicionar a maca ao lado do paciente e auxiliá-lo a deitar em movimentos coordenados conforme técnica de autoextricação que deverá estar descrita em protocolo institucional. O uso do dispositivo de extricação de kendrick (KED) não é mais indicado, pois, leva tempo para ser colocado e dá a falsa sensação de imobilização (COWLEY A, et al., 2017; WHITE CC, et al., 2014; STANTON D, et al., 2017; BROWN N, 2015; DIXON M, et al., 2014; FISHER PE, et al., 2018).

Já em vítima instável sentada na cena e/ou no automóvel, colocar inicialmente o colar cervical e realizar técnica de retirada rápida com três profissionais, colocando a vítima na prancha rígida, conforme protocolo institucional e proceder a RMC vertebral (CONNOR D, et al., 2013; STANTON D, et al., 2017).

Após a construção da proposta do protocolo de RMC vertebral, bem como a definição dos critérios de decisão e das técnicas a serem utilizadas, foi realizada a apresentação destes para a gerência e profissionais do serviço e se realizou alguns ajustes no protocolo.

Está previsto a discussão com a rede de urgência do município para um alinhamento com os serviços que atendam vítimas de trauma e assim, construir um planejamento para iniciar o treinamento de todos profissionais, a implantação do protocolo e monitoramento do mesmo.

\section{DISCUSSÃO}

White CC, et al. (2014) e Fischer PE, et al. (2018), afirmam que estudos apoiam o uso de técnicas de restrição da coluna, mas estas não devem ser aplicadas a todos os casos de trauma de maneira rotineira, como era feito no passado. Provavelmente, a influência de evidências historicamente pobres foi o principal motivo para continuar a imobilização padrão e os protocolos dos serviços de emergências atuais são baseados em práticas históricas ao invés de evidências científicas.

Kornhall DK, et al. (2017) e Maschmann C, et al. (2019), recomendam a RMC vertebral minimalista para as vítimas que apresentam alguma instabilidade identificada na avaliação primária, porém, observou-se que a restrição minimalista não foi uma descrição clara nestes estudos, não demostrando como deve ser feito e com quais recursos. Um estudo apenas citou que a restrição deveria ser feita associando medidas de estabilização que não atrasem o transporte como exemplo manter o controle manual da cervical.

Considerando a realidade brasileira em que se trabalha com vários fatores dificultadores tais como: 0 número de profissionais insuficientes, as condições das vias de trânsito inadequadas e a gravidade das vítimas, pode-se induzir os profissionais a erros e consequente risco de eventos adversos relacionados a 
manipulação inadequada da coluna vertebral e consequente lesão medular. Deste modo, definiu-se para este protocolo que vítimas com "instabilidade na avaliação primária”, está indicada a RMC vertebral de forma que não atrase o tratamento.

Para as vítimas de trauma contuso, foram estabelecidos critérios pelo grupo considerando a realidade do serviço e também optou-se pela utilização da regra canadense (Canadian C-spine Rule - (CCR)) para avaliação da coluna cervical que é um instrumento validado e largamente utilizado no intra-hospitalar, assim como os critérios National Emergency X-Radiography Utilization Study (NEXUS) que incluem a avaliação do nível de consciência, a presença de dor na coluna, de deformidade em coluna, de sintomas neurológicos, de presença de lesões que causam distração, de idade maior que 65 anos, dentre outros (STIELL IG, et al., 2001; STIELL IG, et al., 2003; FISCHER PE, et al., 2018; STANTON D, et al., 2017).

No item "avaliação do nível de consciência”, o grupo incluiu não só avaliação do Glasgow da vítima, como também o uso de álcool e/ou drogas e qualquer barreira de comunicação como língua estrangeira ou deficiência auditiva.

Para os pacientes com indicação de RMC vertebral os autores optaram pela necessidade do uso da RMC vertebral com uso de colar cervical rígido e restrição de toda a coluna mantendo alinhamento da cabeça, cervical e tronco, podendo elevar a cabeceira em até 30ํse necessário (FISCHER PE, et al., 2018).

O item "cinemática de alta velocidade" no critério de decisão "dinâmica do trauma", gerou uma preocupação, pois, os estudos utilizados definem como referência para a realização da RMC vertebral, a velocidade igual ou maior a $100 \mathrm{Km} / \mathrm{h}$. Estes estudos foram realizados em países de primeiro mundo, locais em que as condições das vias de trânsito e dos veículos, não se equiparam a realidade brasileira, fato já relatado anteriormente. Assim, optou-se por não utilizar como referência a velocidade definida nesses estudos e nem estabelecer uma velocidade devido à falta de evidências cientificas que se adequem a realidade do Brasil. Assim em "dinâmica do trauma" o item "qualquer outra situação de trauma que a equipe considere importante a RMC vertebral" foi incluído dando autonomia aos profissionais de avaliar a necessidade de RMC vertebral ou não.

No tocante as vítimas de trauma penetrante, estudos realizados relatam que não existe benefícios de imobilização da coluna vertebral no ambiente pré-hospitalar, pois a taxa de mortalidade duplica em consequência do tempo gasto para realizar a imobilização. Assim, levando em consideração que o tempo é o aspecto mais crítico do tratamento para estes pacientes a imobilização é contraindicada (VELOPULUS CG, et al., 2017; BROWN JB, et al., 2009; SEPPA N, 2010; HAUT E, et al., 2010).

Por fim, este relato apresenta a construção de um protocolo de RMC vertebral que foi elaborado a partir de evidências científicas e particularidades do serviço onde está sendo implantado, o que permite entender que esse protocolo pode ou não ser adequado a outros locais, porém, traz reflexões e uma proposta de atendimento que pode ser adaptada.

Acredita-se que com a construção deste protocolo os serviços pré-hospitalares do Brasil possam iniciar mudanças no tratamento das vítimas de trauma com melhores cuidados baseados em evidências científicas na tentativa de promover uma assistência de qualidade e segura para o paciente. A adoção da RMC vertebral elimina o uso desnecessário de imobilização vertebral que irá, por sua vez, diminuir o tempo para o tratamento definitivo destas vítimas, além de diminuir tempo de liberação da equipe do atendimento.

\section{REFERÊNCIAS}

1. BROWN J, et al. Prehospital immobilization of the spine does not appear to be beneficial and can complicate care after a bullet injury to the trunk. The Journal of Trauma, 2009; 67(4): 774-778.

2. BROWN N. Should the Kendrick Extrication Device have a place in pre-hospital care? Journal Paramedic Practice, 2015; 7(6): 300-4.

3. CONNOR D, et al. Pre-hospital spinal immobilisation: an initial consensus statement. Emergency Medicine Journal, 2013; 30(12): 1067-9.

4. COWLEY A, et al. Cervical spine immobilization during extrication of the a wake patient: a narrative review. European Journal of Emergency Medicine, 2017; 24: 158-161. 
5. DIXON M, et al. Biomechanical analysis of spinal immobilisation during prehospital extrication: a proof of concept study. Emergency Medicine Journal, 2014; 31: 745-749.

6. FISCHER PE, et al. Spinal Motion Restriction in the Trauma Patient - A Joint Position Statement. Prehospital Emergency Care, 2018.

7. HAM WHW, et al. Pressure ulcers, indentation marks and pain from cervical spine immobilization with extrication collars and headblocks: An observational study. Journal Trauma Acute Care Surgery, 2016; 76 (4): 1131-41.

8. HAUT E, et al. Spine immobilization in penetrating trauma: more harm than good? Journal Trauma, 2010 ; 68 (1): 115121.

9. HASLER RM, et al. Epidemiology and predictors of spinal injury in adult major trauma patients: European cohort study. European Spine Journal, 2011; 20 (12): 2174-80.

10. KORNHALL DK, et al. The Norwegian guidelines for the prehospital management of adult trauma patients with potential spinal injury. Scandinavian Journal of Trauma, Resuscitation and Emergency Medicine, 2017; 25:2.

11. MASCHMANN C, et al. New clinical guidelines on the spinal stabilisation of adult trauma patients - consensus and evidence based. Scandinavian Journal of trauma, ressucitation and Emergency Medicine, 2019; 27:77.

12. PHTLS. PREHOSPITAL TRAUMA LIFE SUPPORT. Artmed. 9ª edição. National Association of Emergency Medical Technicians (NAEMT). 2018. 786 pág.

13. PURVIS TA, et al. The definite risks and questionable benefits of liberal pre-hospital spinal immobilisation. American Journal of Emergency Medicine, 2017; (35): 860-866.

14. RAO PJ, et al. Cervical spine immobilization in the elderly population. Journal Spine Surgery, 2016; 2(1): 41-46.

15. SEPPA, N. Gunshot Victims Have Need for Speed: Delay for Spine Stabilization Doubles Death Risk, Study Finds. Science News, 2010; $177(3): 8$.

16. STANTON D, et al. Cervical collars and immobilisation: A South African best practice recommendation. African Journal of Emergency Medicine, 2017; (7): 4-8.

17. STIELL IG, et al. The Canadian C-spine rule for radiography in alert and stable trauma patients. JAMA, 2001; 286(15): 1841-8.

18. STIELL IG, et al. The Canadian C-Spine Rule versus the NEXUS Low-Risk Criteria in Patients with Trauma. New England Journal of Medicine, 2003; 349(26): 2510-8.

19. STIEL IG, et al. The OPALS Major Trauma Study: impact of advanced life-support on survival and morbidity. Canadian Medical Association Journal, 2008; 178(9): 1141-52.

20. SUNDSTROM T, et al. Prehospital use of cervical collars in trauma patients: a critical review. Journal of Neurotrauma, 2014; 31(6): 531-40.

21. VAILLANCOURT C, et al. The Out-of-Hospital Validation of the Canadian C-Spine Rule by Paramedics. Annals of Emergency Medicine, 2009; 54: 663-671.

22. VELOPULOS CG, et al. Prehospital spine immobilization/spinal motion restriction in penetrating trauma: A practice management guideline from the Eastern Association for the Surgery of Trauma (EAST). Journal Trauma Acute Care Surgery, 2017; 84(5): 736-744.

23. WHITE CC, et al. EMS spinal precautions and the use of the long backboard-resource document to the position statement of the National Association of EMS Physicians and the American College of Surgeons Committee on Trauma. Prehospitalar Emergency Care, 2014; 8(2): 306-14. 